\title{
Synthèse
}

\section{État de la lutte dirigée contre les insectes ravageurs en production biologique de pommes de terre}

\author{
Gilles Boiteau $^{1}$ \\ ${ }^{1}$ Centre de recherche en pomme de terre, \\ Agriculture, \\ C.P. 20280 , \\ E3B 4 Z7 Fredericton, \\ Canada \\ <boiteaug@agr.gc.ca>
}

\begin{abstract}
Résumé
La stratégie de lutte dirigée contre les insectes en production biologique est ancrée sur l'utilisation des méthodes de lutte préventives plutôt que curatives. Pour se faire, les producteurs de pommes de terre biologiques du Canada, des États-Unis et de l'Union européenne ont recours aux mêmes méthodes agronomiques naturellement acceptables pour lutter contre les insectes phytoravageurs : la rotation des cultures, l'isolement des champs, la plantation retardée, la gestion des sols et la pose de paillis. Malheureusement, parce que chaque méthode a une efficacité optimale contre une espèce particulière de ravageur, l'intégration de ces méthodes, entre elles ou contre plusieurs espèces, reste plutôt rare et limitée à un seul champ plutôt qu'à la ferme. De plus, la conservation et les lâchers d'agents de lutte biologique sont rarement utilisés en production de pommes de terre parce qu'on sait peu de chose sur leur efficacité, que les ressources manquent ou qu'ils ne sont pas disponibles sur le marché. La demande croissante des consommateurs, pour les aliments biologiques, force les producteurs à faire des choix difficiles. L'accroissement des superficies nécessaires pour répondre à la demande future en pommes de terre biologiques éloigne cette industrie de l'utilisation des méthodes préventives et augmente sa dépendance sur les méthodes curatives. Par exemple, les fermes qui viennent de passer de l'agriculture classique à l'agriculture biologique bénéficient de la tolérance des plantes aux insectes, un atout important des fermes biologiques établies depuis longtemps, et doivent se tourner vers les méthodes curatives.
\end{abstract}

Mots clés : certification; lutte biologique ; lutte physique ; ravageur des plantes ; Solanum tuberosum.

Thèmes : pathologie ; productions végétales ; systèmes agraires.

\section{Abstract \\ Status of insect pest management in organic potato production.}

The insect pest management program in organic potato production gives priority to preventive control methods rather than curative methods. To do so, organic potato growers in Canada, the USA or the European Union rely on similar cultural methods to control insect pests: crop rotation, field isolation, delayed planting, soil management and mulching. Unfortunately, because each method is effective against a particular insect pest, the integration of methods or against multiple pests remains rare and limited to single fields rather than the whole farm. Furthermore, conservation and releases of biological control agents are rarely used in potato production because of limited knowledge on their efficacy, the lack of resources or the unavailability of the control agents on the market. The increasing consumer demand for organically grown food is forcing growers to make difficult decisions. The increase in acreage required to meet future demand is drawing insect pest protection further away from its primary reliance on preventive pest control methods towards a dependence on curative control methods. For example, farms in transition from conventional to organic benefit little from the crop tolerance to insect pests in established long-term organic potato farms and must turn to reactive control methods.

Key words: biological control; certification; physical control; plant pests; Solanum tuberosum.

Subjects: farming systems; pathology; vegetal productions. 
es perspectives de croissance n'ont jamais été meilleures en agriculture biologique. La demande a augmenté si rapidement qu'elle dépasse l'offre (Anonyme, 2006). La culture de la pomme de terre est l'une des nombreuses cultures ayant bénéficié de cet essor, mais elle forme encore une petite proportion de l'agriculture biologique au Canada, aux États-Unis et dans l'Union européenne. En 2005, la pomme de terre couvrait moins de 0,1\% (497 ha) de la superficie totale (Macey, 2006) et représentait 1,5\% des 3618 agriculteurs biologiques certifiés au Canada. La même année, aux États-Unis, 0,16 \% (2 663 ha) de la superficie certifiée biologique (1 640769 ha) produisait des pommes de terre (USDA, 2006). En 2002, en Europe, 5,1 millions d'hectares étaient consacrés à la production biologique. La France, qui arrivait au $14^{\mathrm{e}}$ rang sur 18 pays, avait un peu plus de 400000 ha en production biologique (Yussefi et Willer, 2004) dont 579 ha consacrés à la production biologique de pommes de terre (PAN Europe, 2007). Aux Pays-Bas, les pommes de terre biologiques couvraient $15,14 \%$ de la superficie biologique totale (PAN Europe, 2007). La production à grande échelle de pommes de terre biologiques fait face à plusieurs défis dont la protection contre les insectes ravageurs, ce qui explique le très petit nombre de producteurs ayant de grandes superficies.

En production biologique, le respect d'une philosophie de gestion valable sur le plan biologique ou d'un système préventif régi par des lignes directrices rigoureuses remplace la dépendance à l'égard des méthodes de lutte contre les insectes nuisibles réactives ou curatives, qui caractérisent la production classique de pommes de terre. Historiquement, les concepts de la lutte dirigée sont apparus le long d'un continuum représentant une utilisation décroissante des intrants (Boiteau, 1991): agriculture classique, lutte intégrée, protection durable et, finalement, la protection biologique des pommes de terre. Il s'agit là des étapes d'un système de protection et de production de plus en plus naturel. À une extrémité, la production classique repose sur une seule méthode réactive : les insecticides. À l'autre extrémité, la production biologique s'appuie plus sur des méthodes préventives que curatives. La trousse à outils s'est diversifiée et requiert son propre modèle. Wyss et al. (2005) ont organisé ces outils selon un processus graduel pour la lutte contre les arthropodes dans les vergers, processus qui s'adapte bien aux cultures biologiques en général (Zehnder et al., 2007): (1) les pratiques agronomiques compatibles avec les pro- cessus naturels, (2) la gestion de la végétation visant à favoriser les ennemis naturels, (3) le lâcher d'agents de lutte biologique et (4) les insecticides biologiques approuvés (figure 1). La priorité est accordée aux outils de prévention, puis à des méthodes plus directes. Cette stratégie de lutte dirigée peut être mise en œuvre au sein d'un programme de lutte intégrée à quatre niveaux de complexité (échelle de lutte intégrée de Prokopy (1993)): (1) une seule espèce de ravageur, (2) tous les ravageurs, (3) tous les ravageurs et tout le système de production et (4) intérêts économiques et politiques (figure 1). Enfin, les outils et le niveau d'intégration choisis peuvent être appliqués à l'une des quatre dimensions du paysage: (1) le champ, (2) la ferme, (3) la région agricole et (4) le pays et la planète (figure 1).

Les questions réglementaires sont les principaux facteurs limitant la croissance de l'industrie des pommes de terre biologiques à l'heure actuelle, mais les problèmes de lutte contre les phytoravageurs, comme le ver fil de fer et le doryphore, peuvent représenter des déterminants importants (Tréhoret et Jouan, 2001). Les altises, les cicadelles, les punaises ternes et les pucerons peuvent aussi endommager les récoltes. Les pucerons colonisateurs ou non colonisateurs peuvent en

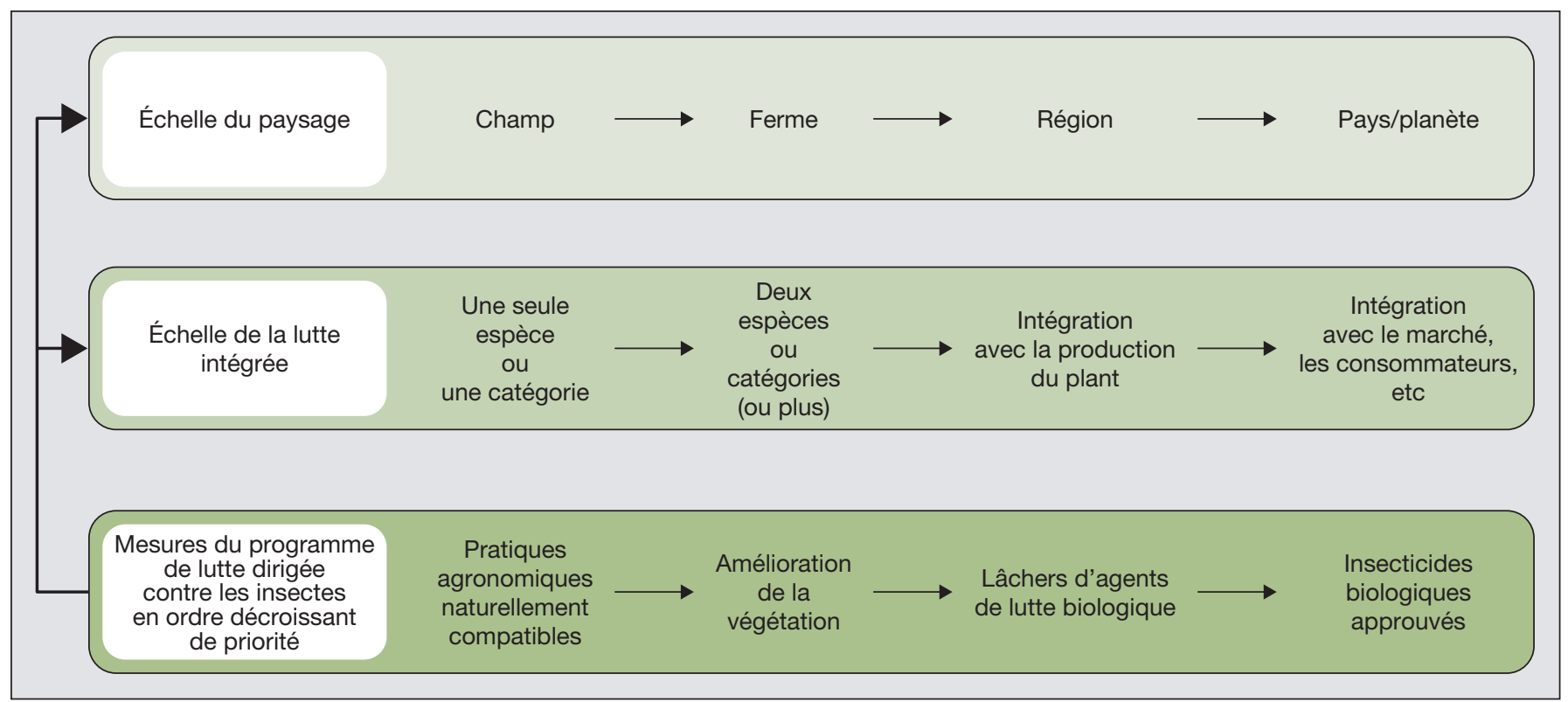

Figure 1. Représentation schématique d'une stratégie de lutte dirigée biologique contre les insectes constituée de quatre ensembles de mesures de lutte de priorité décroissante pouvant chacun être appliqué à un des quatre niveaux croissants d'intégration et à une des quatre dimensions croissantes du paysage (adaptée de Prokopy (1993) et de Wyss et al. (2005)).

Figure 1. Diagrammatic representation of an organic insect pest management program made up of four sets of control measures of decreasing priority that can each be implemented at one of the four increasing levels of integration and at one of the four increasing landscape levels (adapted from Prokopy (1993) and Wyss et al. (2005)). 
outre transmettre des phytovirus ayant des incidences graves sur la production de pommes de terre de semence.

Cet article examine l'applicabilité des quatre mesures de lutte dirigée contre les insectes identifiées par Wyss et al. (2005) à la production biologique de pommes de terre en Amérique du Nord et en Europe et met en évidence les défis qu'il y a à relever.

\section{Pratiques agronomiques naturellement compatibles}

La lutte contre les arthropodes nuisibles en agriculture biologique donne la priorité aux pratiques agronomiques compatibles avec les processus naturels (figure 1): rotation des cultures, emplacement du champ et de la ferme, fertilité du sol, calendrier des pratiques culturales, barrières, résistance de la plante hôte non transgénique. Ces pratiques font partie d'un plan à long terme le long de l'échelle de la lutte intégrée et, bien qu'elles puissent subir des influences à l'échelle régionale ou nationale, s'appliquent à celle du champ ou de la ferme.

L'emplacement du champ de pommes de terre doit convenir le moins possible aux insectes ravageurs. La petite superficie consacrée aux pommes de terre biologiques permet aux agriculteurs de faire pousser leur récolte dans des champs qui satisfont aux exigences agronomiques des plantes tout en étant isolés des ravageurs. Malheureusement, cette situation ne demeurera possible que tant que cette production restera limitée : la demande et la superficie augmentant, cette flexibilité disparaitra et les exigences agronomiques auront priorité.

La rotation des cultures est essentielle pour lutter contre le doryphore de la pomme de terre, dont les adultes passent l'hiver dans les champs de pommes de terre ou dans la lisière forestière avoisinante. Le succès de cette pratique est proportionnel à la distance entre les rotations. Le manque de disponibilité des terres limite l'utilisation de cette pratique (Sexson et Wyman, 2005). La rotation est efficace, mais des insecticides biologiques approuvés seront nécessaires en complément pour lutter contre la larve si les distances de rotation sont courtes. La rotation est efficace aussi contre des ravageurs secondaires comme la pyrale du maïs (Ostrinia nubilalis) mais est inefficace contre la cicadelle de la pomme de terre (Empoasca fabae), sauf dans les régions où elle passe l'hiver.

Dans le cas du ver fil de fer, le plan de rotation et la gestion de la fertilité du sol peuvent, en fait, favoriser leurs populations (Keiser et al., 2005). Les populations élevées de vers fil de fer dans le sol sont en général associées à des champs depuis longtemps en prairie (Ute et Paffrath, 2003). Malheureusement, les cultures qui attirent le ver fil de fer sont souvent les plus économiques à cultiver avant les pommes de terre. Le labourage et le hersage endommagent physiquement les œufs et les larves fil de fer et les exposent aux faibles températures et à leurs ennemis naturels (Ute et Paffrath, 2003). Si le champ infesté est labouré peu avant la plantation plutôt que l'automne précédent, le gazon en décomposition servira de source de nourriture de remplacement pour les vers fil de fer. Dans les régions où il est possible d'attendre que le sol soit réchaufféavant de planter les pommes de terre, les larves auront eu le temps de s'enfouir davantage dans le sol frais qu'elles préfèrent. La récolte hâtive est recommandée aux États-Unis (Hoy et al., 2008) et en Irlande (Ute et Paffrath, 2003, 2004). L'augmentation récente des dégâts au Royaume-Uni (Parker, 2004), dans de nombreuses provinces canadiennes (Vernon, obs. pers.) et dans les cultures classiques et biologiques en Autriche (Brunner et al., 2005), parfois attribuée à la disparition des résidus d'insecticides organochlorés dans le sol des fermes classiques, ne permet pas d'expliquer les dommages observés dans les fermes biologiques.

Les procédures sanitaires qui détruisent les refuges, l'enfouissement des mauvaises herbes et la destruction des zones herbeuses avoisinant le champ sont des pratiques agronomiques traditionnelles pour contrôler les altises mais qui ne peuvent pas être utilisées régulièrement parce qu'elles sont incompatibles avec les pratiques culturales qui s'efforcent de maintenir une couverture sur le sol. Lors de la floraison de la pomme de terre, lutter contre les mauvaises herbes et faucher la végétation peut favoriser les déplacements des punaises ternes vers les parcelles de pomme de terre (Caldwell et al., 2006)
La rotation et la récolte hâtive des pommes de terre de semence réduisent les risques de transmission de virus par les pucerons. Le paillage permet de fortement réduire les populations du doryphore de la pomme de terre en perturbant la dispersion au sol des individus (Stoner et al., 1996). Il réduit aussi l'abondance des pucerons et l'incidence du potyvirus Y dans les régions où les vols de vecteurs se produisent avant la fermeture du couvert (Doring et al., 2006).

Lorsqu'il est impossible de cultiver la pomme de terre loin des insectes ravageurs, des méthodes d'exclusion peuvent être envisagées. Des tranchées dont le sol est recouvert de plastique réduisent la colonisation par le doryphore de $50 \%$ et l'oviposition de $70 \%$ (Boiteau et Vernon, 2000).

Les aspirateurs mécaniques peuvent être efficaces, mais il est nécessaire d'améliorer leur conception pour minimiser les besoins en énergie et le compactage des sols (Vincent et Boiteau, 2000). Le brûleur au propane, plus léger, peut aider à supprimer les doryphores adultes se trouvant sur les plantes et jusqu'à $30 \%$ des œufs (Duchesne et al., 2000).

Selon l'emplacement géographique, la plantation et la récolte hâtive peuvent réduire l'abondance de la cicadelle de la pomme de terre, des pucerons ou du ver fil de fer (Christen et al., 2006). La brièveté de la saison de végétation de nombreuses régions où la pomme de terre est cultivée limite l'utilisation de ces pratiques, qui souffrent de l'absence de variétés de saison courte. Aucune variété de pommes de terre n'est résistante au ver fil de fer (Parker, 2004) ni au doryphore. Certaines variétés sont considérées comme moins sensibles que d'autres à la cicadelle (Caldwell et al., 2006).

Selon Phelan et al., (1996), les plantes poussant dans les sols des fermes biologiques atteignent un équilibre minéral naturel qui leur confère tolérance ou résistance aux insectes ravageurs. Des recherches récentes ont montré que les pommes de terre cultivées dans des sols amendés au fumier entravent le développement du doryphore, par comparaison à des pommes de terre cultivées dans des sols fertilisés aux engrais de synthèse (Alyokhin et Atlihan, 2005). On ne sait pas encore bien si l'effet provenait de l'utilisation de fumier ou de l'application à long terme des pratiques de gestion biologiques.

En pratique, quelques-unes seulement des méthodes mentionnées ci-dessus 
sont utilisées par les producteurs biologiques. Les méthodes telles que les tranchées, le paillage, les cultures-pièges, les brûleurs au propane et les aspirateurs sont en pratique peu utilisées, comme c'est le cas en production classique, au profit de substances autorisées, en raison de leur efficacité relativement faible, du temps et des ressources qu'elles exigent ou de leurs incidences pouvant être négatives sur la santé de la récolte, etc.

\section{Gestion \\ de la végétation}

L'absence d'insecticides de synthèse accroit naturellement la contribution des populations de parasites et de prédateurs indigènes à la lutte contre les insectes nuisibles par rapport à ce qui se produit en production classique de pommes de terre et crée des conditions favorables à leurs activités. L'amélioration de la végétation pour la conservation des agents de lutte biologique accroît la résistance de la culture aux ravageurs mais, lorsque celle-ci est rompue, des méthodes de lutte curatives, comme le lâcher massif d'agents de lutte biologique, sont nécessaires (figure 1). Les avantages de l'amélioration de la végétation ne peuvent être mesurés que comme un investissement à long terme et doivent être évalués en fonction des effets négatifs. Par exemple, les brises vent autour des champs augmentent les populations de prédateurs généralistes mais favorisent également l'hivernage des insectes ravageurs, comme le doryphore.

Les cultures-pièges de pommes de terre plantées plus tôt que la récolte principale pour arrêter les doryphores adultes colonisateurs au printemps, ou ceux qui migrent vers les lieux d'hivernage à la fin de la saison, n'ont remporté qu'un succès moyen en production classique de pommes de terre et exigeaient l'utilisation combinée d'un insecticide pour ne pas que les insectes s'avancent davantage dans le champ ou les lieux d'hivernage (Ferro, 1995; Caldwell et al., 2006). Des bordures de soja ou de pommes de terre peuvent réduire l'incidence du virus $\mathrm{Y}$ chez les pommes de terre de semence (Fereres, 2000).

Les cultures-pièges et les cultures de bordure s'appliquent à l'échelle du champ, mais une bonne partie des méthodes de gestion de la végétation visant à conser- ver les agents de lutte biologique doivent être envisagées à l'échelle régionale ou à celle de la ferme pour être efficaces (figure 1). Leur utilisation ne peut être envisagée que pour certains ravageurs et leur mise en ouvre doit être intégrée à la production agricole. Dans l'ensemble, les connaissances nécessaires pour intégrer ces méthodes sont à ce jour insuffisantes.

\section{Lâchers massifs d'agents de lutte biologique}

Le lâcher d'agents de lutte biologique est une pratique saine sur le plan environnemental mais curative plutôt que préventive parce qu'elle s'applique à une seule espèce de ravageurs et à un seul champ (figure 1).

Les lâchers massifs d'Edovum puttleri, une petite guêpe qui parasite les œufs de doryphore (Sears et Boiteau, 1989) mais surtout de punaises prédatrices (Biever et Chauvin, 1992), pourraient être aussi efficaces que les insecticides de synthèse. Malheureusement, alors que les installations d'élevage ont relevé le défi consistant à produire et à vendre les agents de lutte biologique dont les productions sous serre ont besoin pour effectuer des lâchers massifs, elles n'ont pas réussi à produire le grand nombre d'agents de lutte biologique nécessaires pour traiter les productions en plein champ, comme les pommes de terre. L'instabilité de la demande pour ces agents biologiques empêche l'établissement des installations d'élevage de masse nécessaires. L'utilisation d'agents de biolutte en agriculture biologique doit de plus être approuvée à l'échelle nationale ou internationale, selon le marché cible. La rigueur des exigences des organismes d'homologation biologique partout dans le monde ainsi que le marché relativement restreint offert par la production de pommes de terre continueront de limiter considérablement l'importance de cette stratégie.

\section{Insecticides biologiques approuvés}

En principe, les producteurs biologiques ne devraient pas utiliser de substances autorisées à moins que les méthodes préventives et les agents biologiques n'aient échoué à empêcher que les populations d'insectes ravageurs dépassent des seuils acceptables. En pratique, une industrie en plein essor se consacre à la mise au point, à la production et à la commercialisation de substances de lutte. Dans le cas de la pomme de terre, l'inventaire des substances permises pour la lutte contre les insectes est encore très limité quel que soit le pays considéré (tableau 1).

L'évaluation d'une souche canadienne du pathogène fongique Metarbizium anisopliae, utilisé seul ou associé au spinosad (Ericsson et al., 2007) comme moyen de lutte biologique contre le ver fil de fer, est prometteuse, mais son usage n'a pas été évalué directement en relation avec la production de pommes de terre biologiques. Des formulations commerciales de nématodes parasites (Heterorhabditis et Steinernema) ont aussi été proposées contre le doryphore (Kuepper, 2003). Il est délicat de déterminer si des agents entomopathogènes conviennent mieux que les prédateurs et les parasites. L'insecticide bactérien Bacillus thuringiensis, par exemple, a au début été utilisé avec succès contre le doryphore de la pomme de terre mais a, par la suite, été enlevé de la liste des substances autorisées en raison de la formulation inappropriée des produits.

Cette stratégie de lutte dirigée biologique de dernier recours dans le modèle de production biologique met les bases du mouvement biologique à rude épreuve et amène la production de pommes de terre biologiques de plus en plus près du système de production classique des pommes de terre.

\section{Conclusion}

Les producteurs de pommes de terre biologiques sont sensibles à leur environnement et sont donc déterminés à produire des récoltes saines de façon durable. Ils mettent l'accent sur la relation entre la santé de la culture et la qualité du sol. La santé des plantes produites biologiquement contribue, en effet, à leur résistance aux insectes. Cela ne fait cependant que supplémenter le rôle des autres méthodes de lutte biologique dirigée telles que la rotation des cultures, la pose de paillis ou la plantation retardée. Ces pratiques agronomiques naturellement compatibles 


\section{Tableau 1. Liste sommaire des insecticides biologiques approuvés utiles en production de pommes de terre}

Table 1. List of biological insecticides approved for use in organic potato production.

\begin{tabular}{|c|c|c|}
\hline Insecticide & Utilisation & Insecte cible \\
\hline Pyréthrines & Autorisés mais limités & Plusieurs ravageurs mais aussi toxique contre les insectes utiles \\
\hline $\begin{array}{l}\text { Azadirachtine } \\
\text { (extrait de neem) }\end{array}$ & Populaire & Plusieurs ravageurs \\
\hline Spinosad & $\begin{array}{l}\text { Canada et États-Unis } \\
\text { moins utilisé en Europe }\end{array}$ & Plusieurs ravageurs mais pas la cicadelle \\
\hline Savon & Populaire & Pucerons et altises non contrôlés par pratiques culturales \\
\hline $\begin{array}{l}\text { Roténone } \\
\text { (certaines formulations) }\end{array}$ & Limité & Doryphores et pucerons \\
\hline Kaolin & Limité & Pucerons (efficacité probable) \\
\hline Extraits d'herbe & Populaire & Plusieurs ravageurs réputation de repousser ou d'inhiber les insectes \\
\hline Beauveria bassiana & Limité & Doryphores (efficacité variable combinaison avec autres produits) \\
\hline
\end{tabular}

Source : (Kuepper, 2003 ; Caldwell et al., 2006 ; Anonyme, 2007 ; USDA, 2007 ; CEE, 2007).

peuvent être très efficaces mais ne sont souvent utilisées en lutte dirigée que si elles permettent d'abord d'améliorer ou de soutenir les rendements. L'amélioration de la végétation est rarement retenue comme moyen de lutte contre les insectes parce que ces méthodes exigent un investissement considérable en temps et en ressources.

Les effets ciblés de certaines pratiques, notamment les lâchers d'agents de lutte biologique, intéressent les producteurs mais ne sont généralement pas disponibles. Les insecticides biologiques deviennent de plus en plus attrayants. Sauf pour quelques produits, le peu d'efficacité de nombreux insecticides approuvés et le désir de respecter la nature obligent cependant plusieurs producteurs à tolérer des populations élevées d'insectes ravageurs et à accepter de faibles rendements (PAN Europe, 2007). Les principes de production étant les mêmes, il y a une très grande similitude des listes de substances autorisées contre les insectes ravageurs de la pomme de terre pour le Canada (Anonyme, 2007), les États-Unis (USDA, 2007) et l'Union européenne (CEE, 2007).

Les normes de base de l'International Federation of Agricultural Movements (IFOAM) pour la production et la transformation (IFOAM, 2005) fournissent les critères fondamentaux utilisés pour la formulation des réglementations nationales. La réglementation peut varier selon les pays, mais la pression d'actualisation et d'uniformisation se fait davantage sentir parce que les lignes directrices interfèrent avec le commerce (USDA, 2007 ; Zehnder et al., 2007 ; Anonyme, 2007 ; CEE, 2007).
Des données sont lentement recueillies sur les avantages à long terme pour l'environnement, et les ressources énergétiques nécessitées la mise en œuvre de stratégies de production biologique à l'échelle des fermes et des régions (Mader et al., 2002). Toutefois, l'utilisation réussie de pratiques agronomiques naturellement compatibles intégrées à la production agricole à l'échelle des fermes et à l'échelle régionale pour protéger les récoltes contre les pertes de rendement causées par les insectes reste un défi à propos duquel il existe peu de renseignements. L'intérêt des consommateurs pour les aliments biologiques place cette industrie en concurrence avec l'industrie classique dite durable ou à intrants réduits. L'écart ou la différence entre les cultures en système de production biologique et en système de production classique diminue. En Europe, par exemple, d'aucuns demandent que des normes de lutte intégrée adaptées à une culture donnée soient établies à l'échelle nationale et que leur application soit obligatoire pour les cultures classiques (PAN Europe, 2007). Une étude récente effectuée dans l'État de Washington (Koss et al., 2005) a révélé que, bien que les densités de prédateurs soient élevées dans les champs biologiques, les densités de pucerons verts du pêcher et de doryphores étaient aussi très élevées. Produire des pommes de terre biologiques en monoculture, avec ou sans usage limité d'insecticides, tout en conservant des rendements élevés, est un défi qui, la superficie augmentant pour répondre à la demande croissante des consommateurs, prendra vraisemblablement une place prépondé- rante. On ne voit pas bien quel sera le partage des débouchés entre la production biologique et la production classique utilisant la lutte intégrée dans laquelle des insecticides sélectifs permettent aux producteurs d'obtenir des densités de prédateurs similaires à celles des champs biologiques tout en conservant les faibles densités de prédateurs caractéristiques des champs traités à l'aide d'insecticides à large spectre (Koss et al., 2005).

L'information sur la lutte contre les phytoravageurs en production biologique de pommes de terre provient en grande partie des pages Web des associations de producteurs biologiques et des fournisseurs de produits de lutte naturelle. Une bonne partie de la démarche en matière de lutte contre les insectes repose sur l'expérience personnelle des producteurs: c'est le fruit des essais et des erreurs. La majorité des constatations de la recherche effectuée sur les fermes classiques peut être transférée aux fermes biologiques, mais l'interaction entre ces conclusions et l'étendue de leurs incidences sur la lutte contre les insectes ne peuvent être déterminées que par des recherches effectuées sur des champs certifiés biologiques.

\footnotetext{
Remerciements

J'aimerais mentionner la contribution de Claude Bertheleme, spécialiste des productions biologiques, ministère de l'Agriculture, des Pêches et de l'Aquaculture du Nouveau-Brunswick, à ce manuscrit.
} 


\section{Références}

Alyokhin A, Atlihan R. Reduced fitness of the Colorado potato beetle (Coleoptera: Chrysomelidae) on potato plants grown in manureamended soil. Environ Entomol 2005; 34 963-8.

Anonyme. The global market for organic food \& drink: business opportunities \& future out look. Report \#7002-40. London : Organic Monitor Ltd., 2006a.

Anonyme. Systèmes de production biologi que: principes généraux et normes de gestion ICS 67.040, norme nationale du Canada. Office des normes nationales du Canada, 2007.

Biever KD, Chauvin RL. Suppression of the Colorado potato beetle (Coleoptera: Chrysomelidae) with augmentative release of predamelidae) with augmentative release of preda$J$ Econ Entomol $1992 ; 85$ : 720-6.

Boiteau G. Integrated insect pest management, sustainable agriculture and the potato crop. In : Proceedings of the Symposium on Sustainable Agriculture: Reducing the Non Renewables. Truro (Nova Scotia, Canada): Nova Scotia Agricultural College, 1991

Boiteau G, Vernon R. Barrières physiques contre les insectes nuisibles. In : Vincent $C$, Panneton B, Fleurat-Lessard $\mathrm{F}$, eds. La lutte physique en phytoprotection. Paris : Inra éditions, 2000.

Brunner N, Kromp B, Meindl P, et al. Evaluation of different sampling techniques for wireworms (Coleoptera : Elateridae) in arable land IOBC/wprs 2005 ; Bulletin 28 : 117-22.

Caldwell B, Brown Rosen E, Sideman E, et al. Resource guide for organic insect and disease management. Geneva (New York, Etats-Unis) : management. Geneva (New York, Etats-Unis) :
New York State Agricultural Experiment Station, 2006.

Communautés économique européenne (CEE). Council regulation $n^{\circ} 2092 / 91$, sur la producCouncil regulation $n^{\circ}$
tion biologique. 2007 .

http ://organicrules.org/ e

http ://www.organic-revision.org/.

Christen C, Paffrath A, Schepl U, et al. Approaches to wireworm control. In : Organic potato
production, sous-groupe "Soil Insect Pests" du groupe de travail "Entomopathogens and entomoparasitic nematodes " de I'OILB-SROP résumés de la $5^{\mathrm{e}}$ réunion, 16 au 18 octobre, Research Centre for Agriculture and Forestry Laimburg (Italie), 2006

Doring $T$, Heimbach $U$, Thieme T, et al. Aspects of straw mulching in organic potatoes II. Effects on potato virus Y, Leptinotarsa decemlineata (Say) and tuber yield. Nachr Dtsch Pflanzenschutzd 2006 ; $58: 93-7$.
Duchesne RM, Lague C, Khelifi M, et al. Répression thermique du doryphore de la pomme de terre. Pp 57-70 In : Vincent C, Panneton B, Fleurat-Lessard F, eds. La lutte physique en phytoprotection. Paris: Inra éditions, 2000.

Ericsson JD, Kabaluk JT, Goettel MS, et al. Spinosad interacts synergistically with the insect pathogen Metarhizium anisopliae against the exotic wireworms Agriotes lineatus and Agriotes obscurus (Coleoptera : Elateridae). J Econ Entomol 2007 ; 100 : 31-8.

Fereres A. Barrier crops as a cultural control measure of non-persistently transmitted aphid-borne viruses. Virus Res $2000 ; 71: 221$ 31.

International Federation of Organic Agriculture Movements (IFOAM). Norms for organic
production and processing: IFOAM basic standards. Bonn (Allemagne) : IFOAM, 2005.

Ferro DN. Mechanical and physical control of the Colorado potato beetle and aphids. In Duchesne RM, Boiteau G, eds. Potato insect pest control proceedings. Potato insect pest control, development of a sustainable approach. Québec : ministère de I'Agriculture des Pêcheries et de l'Alimentation, 1995.

Hoy CW, Boiteau G, Alyokhin A, et al. Managing insect and mite pests. In : Johnson DA ed. Potato health management. Saint-Paul. Minnesota: The American Phytopathological Society, 2008.

Keiser A, Schneider JHM, Haberli M, et al. Influence of farming system, specific cultivation methods and site parameters on potato quality in Switzerland. Actes de la conférence de l'Association européenne pour la recherche sur la pomme de terre, Bilbao, juillet 2005.

Koss AM, Jensen AS, Schreiber A, et al. Comparison of predator and pest communities in Washington potato fields treated with broadspectrum, selective, or organic insecticides. Environ Entomol $2005 ; 34: 87-95$.

Kuepper G. Colorado potato beetle: organic control options. Publication CT107. Fayetteville (Arkansas) : National Sustainable Agriculture Information Service, 2003.

Macey A. Certified organic production in Canada 2005. Ottawa: Canadian Organic Growers Inc, 2006.

Mader PA, Fliebbach D, Dubois L, et al. Soil fertility and biodiversity in organic farming. Science $2002 ; 296$ : 1694-7.

Pesticide Action Network (PAN Europe). Potato production. State of art of integrated crop management/organic systems in Europe, with particular reference to pest management. Communication $\mathrm{n}^{\circ} 8$. London: PAN Europe 2007.

Parker B. Wireworm control on potato. In National potato conference proceedings 2004 Carlow (Ireland): Irish Agriculture and Food Development Authority, 2004.
Phelan PL, Norris KH, Mason JF. Soil management history and host preference by Ostrinianubilalis : evidence for plant mineral balance mediating insect plant interactions. Environ Entomol 1996 ; 25 : 1329-36.

Prokopy RJ. Stepwise progress toward IPM and sustainable agriculture. The IPM Practioner $1993 ; 15: 1-4$

Sears MK, Boiteau G. Parasitism of Colorado potato beetle (Coleoptera: Chrysomelidae) eggs by Edovum puttleri (Hymenoptera : Eulophidae) on potato in eastern Canada. $J$ Econ Entomol $1989 ; 82$ : 803-10.

Sexson DL, Wyman JA. Effect of crop rotation distance on populations of Colorado potato beetle (Coleoptera: Chrysomelidae) : development of areawide Colorado potato beetle pest management strategies. J Econ Entomol $2005 ; 98: 716-24$.

Stoner KA, Ferrandino FJ, Gent MPN, et al. Effects of straw mulch, spent mushroom compost, and fumigation on the density of Colorado potato beetles in potatoes. J Econ Entomol $1996 ; 89$ : 1267-80

Tréhorel F., Jouan B. Pomme de terre bio : une filière embryonnaire dont le développement ne va pas de soi, Agri, 2001; septembre/ octobre, $\mathrm{n}^{\circ} 49$.

United States Department of Agriculture (USDA). Data sets : Organic Production, Economic Research Service, The Economics of Food Farming Natural Resources and Rural America. Washington (DC) : USDA, 2006. www.ers.usda.gov/Data/Organic/.

United States Department of Agriculture (USDA). National Organic Program (NOP), Agricultural Marketing Service. Washington (DC) : USDA, 2007.

www.ams.usda.gov/NOP/NOP/standards/ FullText.pdf.

Ute S, Paffrath A. The development of strategies to regulate the infestation of wireworms (Agriotes spp. L.) in organic potato farming. Beitrag präsentiert bei der Konferenz: Wissenschaftstagung zum Ökologischen Landbau "Ökologischer Landbau der Zukunft", Vienne. 2003.

Vincent C, Boiteau G. Strategies d'utilisation de lutte contre les insectes par aspiration. In : Vincent C, Panneton B, Fleurat-Lessard F, eds. La lutte physique en phytoprotection. Paris : Inra éditions, 2000.

Wyss EH, Luka L, Pfiffner C, et al. 2005. Approaches to pest management in organic agriculture : a case study in European apple orchards, Organic Res 2005 ; mai:33-6.

Yussefi M, Willer $\mathrm{H}$. The world of organic agriculture 2004 : statistics and future prospects. IFOAM Publication, 6th revised edition. Bonn (Germany) : International Federation of Organic Agriculture Movements (IFOAM), 2004.

Zehnder G, Gurr GM, Kuhne S, et al. Arthropod pest management in organic crops. Annu Rev Entomol 2007 ; 52 : 57-80. 